\title{
Vortexlike power flow at the interfaces of metamaterial lens
}

\author{
Kai Fang $^{1}$, Yewen Zhang ${ }^{1 *}$, Liwei Zhang ${ }^{1,2}$ \\ ${ }^{1}$ MOE Key Laboratory of Advanced Micro-structure Materials, Department of Physics, \\ Tongji University, Shanghai, China \\ ${ }^{2}$ School of Physics and Chemistry, Henan Polytechnic University, Jiaozuo, China \\ *corresponding author, E-mail: yewen.zhang@tongji.edu.cn
}

\begin{abstract}
The metamaterial lens with the sandwich structure of one negative refractive index region between two positive permittivity and permeability regions has been realized by using the two-dimensional (2D) isotropic transmission line approach. We studied the vortexlike power flow at the interfaces of metamaterial lens and validated by the finitedifference time-domain (FDTD) simulator. The computational results showing the different conditions near interfaces are obtained by CST STUDIO SUITE at different frequencies, and demonstrate the intuitionistic power localization at the metamaterial lens interfaces.
\end{abstract}

\section{Introduction}

Since the pioneer work of Veselago's paper about lefthanded media in 1968, the studies of metamaterials have intrigued researchers in the last decade mainly due to the remarkable properties, such as negative refraction, perfect lens with imaging resolution beyond the usual diffraction limit [1-3]. Researches confirmed the existences of the perfect imaging with metamaterial lens based on the transmission line and other structures [4-8]. The dispersion relations in the negative index medium over a frequency range are different from that in a conventional medium [9]. With the changing of frequency, the permittivity, permeability and refractive index of metamaterials can be positive, negative and zero, as the phenomena in the phononic crystal depending on the angle of incidence [10]. Metamaterials essentially characterized by negative value of the permittivity, permeability and refractive index result in opposite directions of the wave vector and the Poynting vector. Near the interface between left-handed media and convectional media, the power flow is inevitably vortexlike because of negative refraction. The properties of vortexlike interface mode in one-dimensional metamaterials were studied, and the group delay from the composite right-lefthanded transmission line to the right-handed transmission line was shown much longer because the vortex mode leads to slow propagation [11]. The vortex-like surface modes inducing image oscillation and the linear guided waves with a dipole-vortex internal structure of the Poynting vector in left-handed slab waveguides have been demonstrated $[12,13]$.

\section{Theory}

In this paper, we studied the vortexlike power flow at the different interfaces of two-dimensional metamaterials with sandwich structure shown in Figure 1. In some frequency bands, the two-dimensional microwave metamaterial lens consists of the negative refractive index region with negative permittivity and permeability (DNG), and positive refractive index region with positive permittivity and permeability (DPS) which can be realized by using transmission lines [14,15]. From Maxwell's source free equations, we can get the electric field $\overrightarrow{\mathrm{E}}$ and the magnetic field $\overrightarrow{\mathrm{H}}$, the plane electromagnetic TEM wave in the homogenous media can be written as

$$
\begin{aligned}
& \mathrm{E}_{\mathrm{z}}=\mathrm{E}_{0} \mathrm{e}^{\mathrm{i}\left(\mathrm{k}_{\mathrm{x}} \mathrm{x}+\mathrm{k}_{\mathrm{y}} \mathrm{y}-\omega \mathrm{t}\right)} \\
& \mathrm{H}_{\mathrm{x}}=\mathrm{H}_{0} \mathrm{e}^{\mathrm{i}\left(\mathrm{k}_{\mathrm{y}} \mathrm{y}+\mathrm{k}_{\mathrm{z}} \mathrm{z}-\omega \mathrm{t}\right)}=\frac{\mathrm{k}_{\mathrm{y}}}{\omega \mu} \mathrm{E}_{0} \mathrm{e}^{\mathrm{i}\left(\mathrm{k}_{\mathrm{y}} \mathrm{y}+\mathrm{k}_{\mathrm{z}} \mathrm{z}-\omega \mathrm{t}\right)} \\
& \mathrm{H}_{\mathrm{y}}=\mathrm{H}_{0} \mathrm{e}^{\mathrm{i}\left(\mathrm{k}_{\mathrm{x}} \mathrm{x}+\mathrm{k}_{\mathrm{z}} \mathrm{z}-\omega \mathrm{t}\right)}=-\frac{\mathrm{k}_{\mathrm{x}}}{\omega \mu} \mathrm{E}_{0} \mathrm{e}^{\mathrm{i}\left(\mathrm{k}_{\mathrm{x}} \mathrm{x}+\mathrm{k}_{\mathrm{z}} \mathrm{z}-\omega \mathrm{t}\right)}
\end{aligned}
$$

where the wavevector obeys $\mathrm{k}^{2}=\mathrm{k}_{\mathrm{x}}{ }^{2}+\mathrm{k}_{\mathrm{y}}{ }^{2}=\omega^{2} \mu \varepsilon \cdot \varepsilon$ and $\mu$ refer to the permittivity and permeability in the isotropic media, while $\overrightarrow{\mathrm{E}}$ and $\overrightarrow{\mathrm{H}}$ are functions of position. In the isotropic media, the time average of the Poynting vector is

$$
\langle\overrightarrow{\mathrm{S}}\rangle=\frac{1}{2} \operatorname{Re}\left(\overrightarrow{\mathrm{E}} \times \overrightarrow{\mathrm{H}}^{*}\right)=\frac{\overrightarrow{\mathrm{k}}}{2 \omega \mu}|\overrightarrow{\mathrm{E}}|^{2}=\frac{\overrightarrow{\mathrm{k}}}{2 \omega \varepsilon}|\overrightarrow{\mathrm{H}}|^{2}
$$

We conceptually assume the media to be lossless for simplicity and then, the material parameters are taken to be real-valued quantities. In the DPS region, Poynting vector possesses the same direction with the wave vector because $\varepsilon$ and $\mu$ are positive simultaneously. On the contrary, the Poynting vector changes to the opposite direction of the wave vector in DNG region. The plane wave is the backward wave in the DNG material. Therefore, near the interface between the positive material and the negative material, the changes of Poynting vector direction result in the vortexlike power flow in conditions of the oblique incidence. 


\section{Discussion}

In our metamaterial lens model, the DNG metamaterial region is constructed by periodically isotropic transmission line medium loaded inductors and capacitors, while the DPS one is constructed of the isotropic microstrip grids. And the sample of the metamaterial lens consists of the left DPS region with $5 \times 11$ cells, an adjacent DNG region with $10 \times 11$ cells, and the right DPS region with $11 \times 11$ cells in $(x$, y) plane. The DPS medium and the DNG host medium are designed on a dielectric substrate $\left(\varepsilon_{\mathrm{r}}=4.75\right)$ which height $\mathrm{h}$ is $1.6 \mathrm{~mm}$ with a square grid of $\mathrm{w}=2.8 \mathrm{~mm}$ wide microstrip lines. The unit period $\mathrm{d}$ is $1 \mathrm{~cm}$ in $\mathrm{x}$ and $\mathrm{y}$ directions. Inductances and shunt capacitances are $3.3 \mathrm{nH}$ and $2.5 \mathrm{pF}$ in the DNG region. The schemes are shown in the insets of Figure 1. In the long-wavelength regime, the permittivity and permeability of metamaterials can be artificially synthesized with periodic L-C networks and double negative values with a negative refractive index in the highpass configuration $[14,15]$. Here the unit cell dimensions along both of the $\mathrm{x}$-axis and the $\mathrm{y}$-axis are same and much smaller than a wavelength and these arrays of these unit cells can be viewed as isotropic and effective media.

In the loaded transmission line regions, with the intrinsic dispersions and the effective parameters as mentioned above, we find three different behaviors according to the signs of permittivity, permeability and refractive index as follows: (i) The double negative (DNG) with permittivity, permeability and refractive index $<0$, within the frequency regime of $0.876 \mathrm{GHz}<\mathrm{f}<1.77 \mathrm{GHz}$. Bragg frequency in this structure is $0.876 \mathrm{GHz}$. The focus frequency is $1.25 \mathrm{GHz}$ in the lens which predicts a refractive index of $n=-1$. Within this band, the Poynting vector has opposite direction of wavevector, because the relative permeability and permittivity are negative. (ii) Zero index material (ZIM), with the permittivity, permeability and refractive index in metamaterial are nearly zero while the frequency is about $1.77 \mathrm{GHz}$. Most power flow is reflected at the interface between the DPS region and the ZIM one. (iii) The double positive (DPS) with the permittivity, permeability and refractive index $>0$, when $\mathrm{f}>1.77 \mathrm{GHz}$, and the Poynting vector has same direction with wavevector. The permittivity, permeability and refractive index of metamaterial region are shown in Figure 2 produce positive, negative or zero value at different frequencies. In the combined metamaterial, there are three conditions at different frequencies, such as DPS/DNG/DPS, DPS/DPS/DPS and DPS/ZIM/DPS.

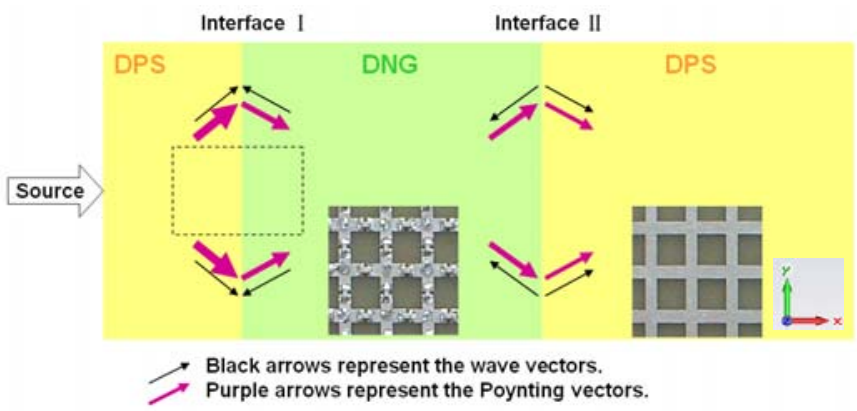

Figure 1: Structure of the metamaterial lens.

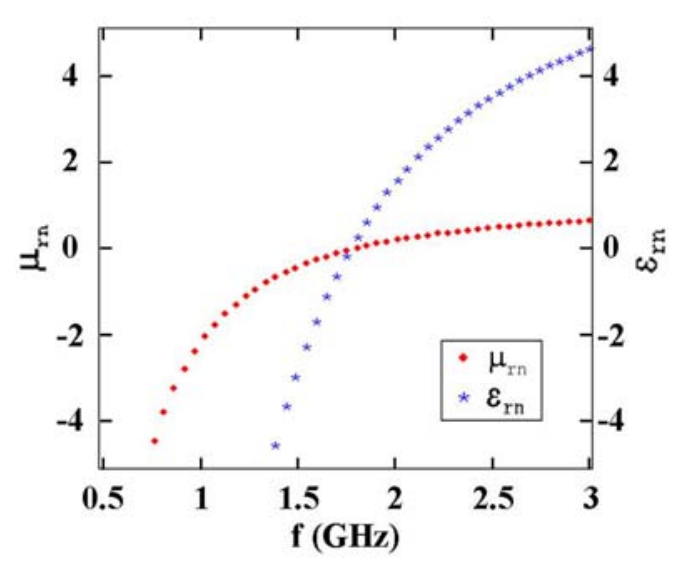

(a) The permittivity and permeability of metamaterial region.

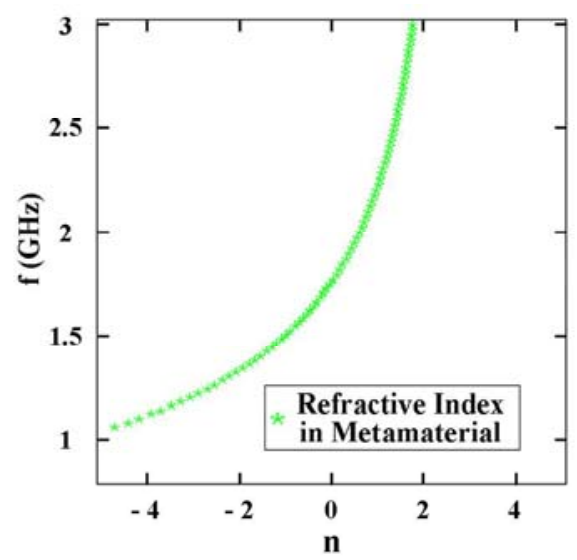

(b) The refractive index of metamaterial region.

Figure 2: The permittivity, permeability and refractive index of metamaterial region.

\section{Simulation}

A time domain simulation was carried out by CST simulator. The Gaussian modulated TEM polarized signal is inputted into the sample at source port shown in Figure 1. From the source port, the wave imported is scattering and the electric fields decay away. With the limitation of the sample size, the oblique incident angle at the interface $\mathrm{I}$ is in the range from -45 degree to 45 degree. On the contrary, in the DNG region, Poynting vector should be pointed away from the source, the phase velocity vector should be pointed toward the source, as depicted in Figure 1.

Figure 3 shows the Poynting vector distribution near the interfaces at different frequencies. The blue dashed lines show the interface between the different regions, and the yellow arrows show the direction of the vortexlike power flow. In Figure 3(a) and (b), the computational results showing the vortexlike power flow near the DPS/DNG and DNG/DPS interfaces are obtained at the focus frequency of $1.25 \mathrm{GHz}$ via metamaterials lens, while the refractive index of DPS and DNG is -1 . At the focus frequency, the wave impedance in DNG region is about $141 \mathrm{Ohm}$, similar to the wave impedance in DPS, while relative permeability and permittivity are about negative one. Therefore, the impedance is matched in different regions of the medium. 
At the interfaces between DPS and DNG media, the direction of the Poynting vector differs considerably from those existing at interfaces between two DPS materials. The direction of Poynting vector in one grating unit is clockwise in the upper part of the region, while the one is anticlockwise in the underside. Similar behaviors show in the other regions near the interface in the condition of oblique incidence.

Contrastively, when the simulation signal frequency is $2 \mathrm{GHz}$, the value of refraction index in the two dimensional loaded transmission lines becomes positive, and the interfaces changes to DPS/DPS. The vortexlike power flow does not take place in this case, shown in Figure 3 (c).

There is a special frequency region, around $1.77 \mathrm{GHz}$, while the permeability, the permittivity and group velocity in the DNG region approach zero. The amplitude of the wavevector in the two dimensional loaded transmission lines is nearly zero. Therefore, the transmission through the interface between the DPS and the ZIM regions is almost forbidden, and the interface becomes a reflecting one, shown in Figure 3 (d).

The vortexlike power flow on the interfaces changes the directions of power flow in the metamaterial lens, and produces the first focus phenomenon in the DNG region and the second focus in the second DPS region mentioned, shown in Figure 4 (a). The change of the vortexlike Poynting vector direction at the DPS/DNG interface is also an important feature that makes the localization for focus in the metamaterial lens. On the contrary, at the DPS/DPS interface, the power flow of electromagnetic wave scatters through the interface and its amplitude decay, shown in Figure 4 (b). Near the interface between the DPS and ZIM regions, most of the wave and the power flow can not pass the interface and be dispersed along the DPS/ZIM interface, shown in Figure 4 (c).

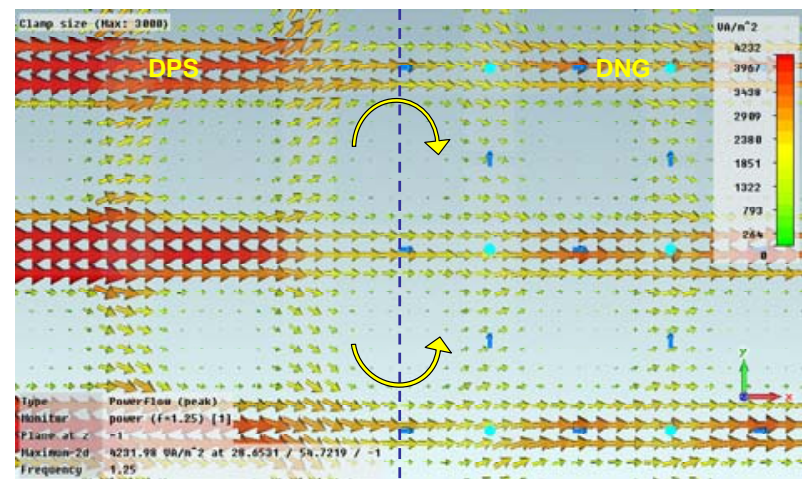

(a) Vortexlike power flow at the DPS/DNG interface in $1.25 \mathrm{GHz}$ near the interface I in the black dashed rectangle region in Figure 1.

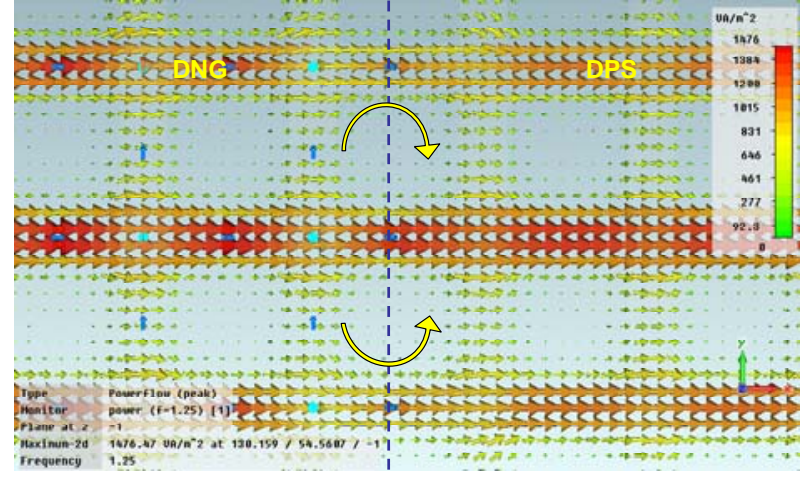

(b) Vortexlike power flow at the DNG/DPS interfaces in $1.25 \mathrm{GHz}$ near the interface II in Figure 1.

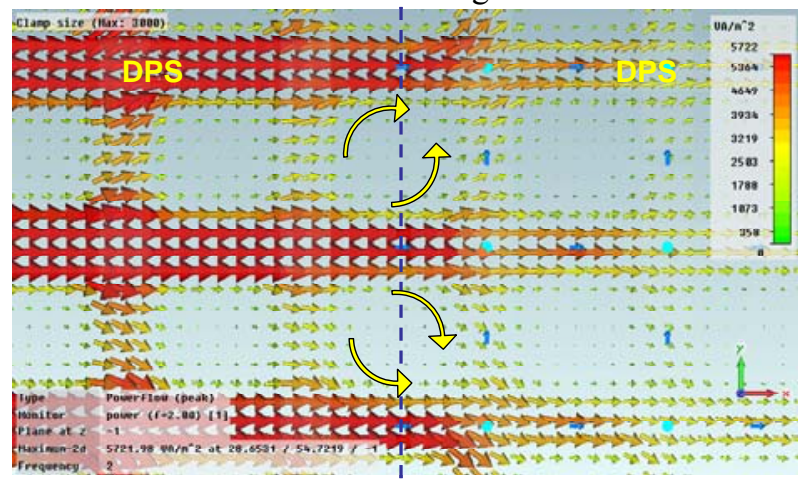

(c) Power flow in $2.00 \mathrm{GHz}$ near the interface $\mathrm{I}$ in the black dashed rectangle region in Figure 1.

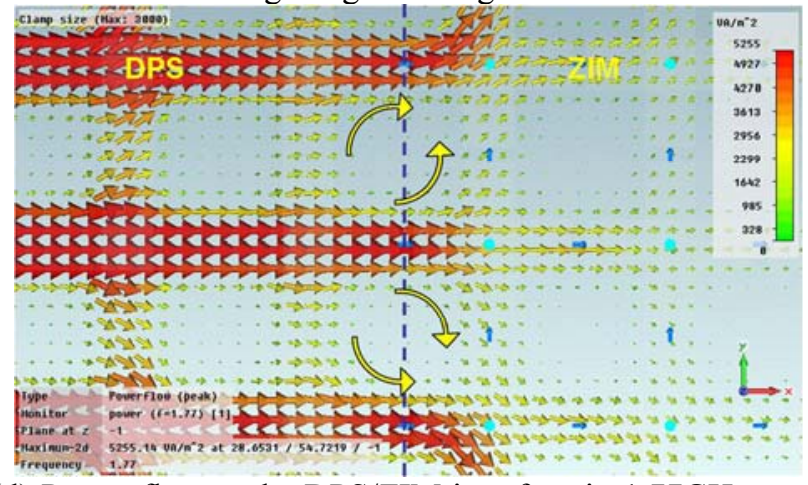

(d) Power flow at the DPS/ZIM interface in $1.77 \mathrm{GHz}$ near the interface $I$ in the black dashed rectangle region in Figure 1, resulting in little transmission.

Figure 3: The power flow at the interfaces of metamaterial with sandwich structure.

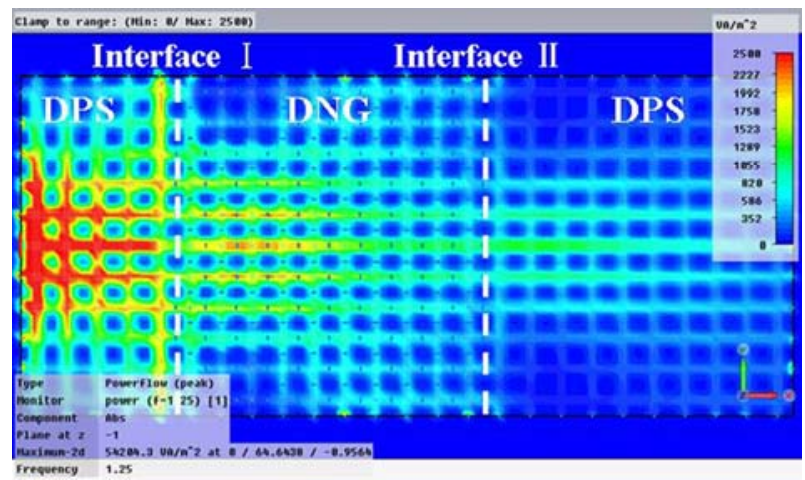

(a) $1.25 \mathrm{GHz}$ 


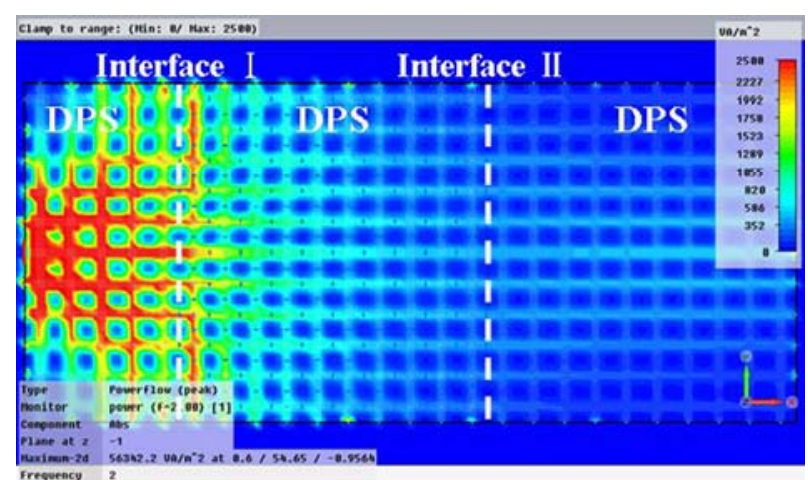

(b) $2.00 \mathrm{GHz}$

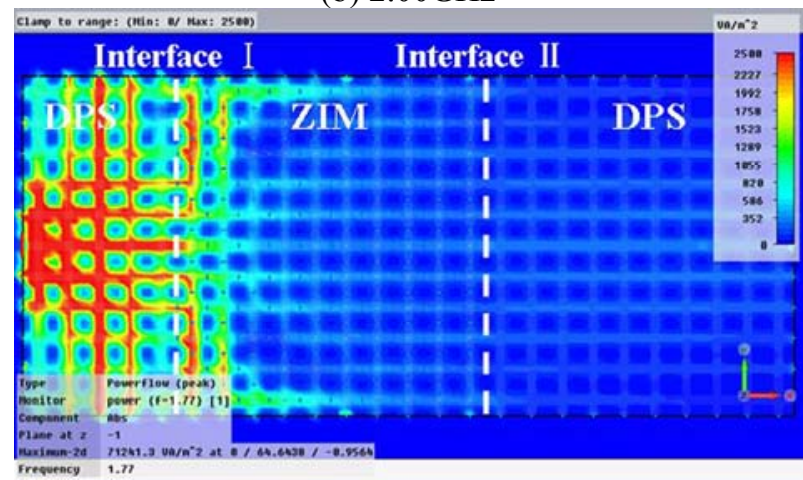

(c) $1.77 \mathrm{GHz}$

Figure 4: Amplitudes of power flow of the sample at different frequencies. (a) Amplitudes of power flow focused in the DNG region and the second DPS region in 1.25GHz. (b) Amplitudes of power flow are decaying away from the interface in $2.00 \mathrm{GHz}$. (c) Amplitudes of power flow in the DPS/ZIM/DPS regions in $1.77 \mathrm{GHz}$.

\section{Conclusion}

To summarize, we have studied the characters of power flow at the interfaces in the metamaterial lens. The energy flow forms vortices at the interfaces between the DPS and DNG regions and the energy was partly trapped. Therefore, the localization at the metamaterial lens interfaces can be explained. The new message in this paper is analysis of the vortexlike power flow and the explanation of the localization at the metamaterial lens interfaces which may have potential applications in metamaterials-based imaging system.

\section{Acknowledgements}

The present research was supported by National Basic Program (973) of China (No.2011CB922001), and by the National Natural Science Foundation of China (Nos. 10634050, 10904032), and by the Excellent Youth Teachers Program of Universities in Henan Province (2012GGJS060).

\section{References}

[1] V. G. Veselago, The electrodynamics of substances with simultaneously negative values of $\varepsilon$ and $\mu$, Sov. Phys. Usp. 10: 509-514, 1968.
[2] J. B. Pendry, Negative refraction makes a perfect lens, Phys. Rev. Lett. 85(18): 3966-3969, 2000.

[3] D. R. Smith, J. B. Pendry, M. C. K. Wiltshire, Metamaterials and negative refractive index, Science 305: 788-792, 2004.

[4] A. Grbic and G. V. Eleftheriades, Overcoming the diffraction limit with a planar left-handed transmissionline lens, Phys. Rev. Lett. 92(11), 117403, 2004.

[5] G. V. Eleftheriades, Negative refraction and focusing in hyperbolic transmission-line periodic grids, IEEE Trans. Microw. Theory Tech. 53(1): 396-403, 2005.

[6] P. Alitalo, S. Maslovski, and S. Tretyakov, Threedimensional isotropic perfect lens based on LC-loaded transmission lines, J. Appl. Phys. 99, 064912, 2006.

[7] T. J. Cui, Q. Cheng, W. B. Lu, Q. Jiang, and J. A. Kong, Localization of electromagnetic energy using a left-handed-medium slab, Phys. Rev. B 71, 045114, 2005.

[8] C. Caloz and T. Itoh, Electromagnetic metamaterials, transmission line theory and microwave applications, Wiley, New York, pp. 133-170, 2006.

[9] G. V. Eleftheriades, K. G. Balmain, Negativerefraction metamaterials: fundamental principles and applications, Wiley, New York, pp.214-219, 2005.

[10] J. Bucay, E. Roussel, J. O. Vasseur, P. A. Deymier, AC. Hladky-Hennion, Y. Pennec, K. Muralidharan, B. Djafari-Rouhani, and B. Dubus, Positive, negative, zero refraction, and beam splitting in a solid/air phononic crystal: Theoretical and experimental study, Phys. Rev. B 79, 214305, 2009.

[11] Y. Wang, Y. Zhang, L. He, H. Li, H. Chen, F. Liu, C. Caloz, Time-domain study of vortexlike interface mode in metamaterials, Appl. Phys. Lett. 91, 221907, 2007.

[12] L. Zhou, and C. T. Chan, Vortex-like surface wave and its role in the transient phenomena of meta-material focusing, Appl. Phys. Lett. 86, 101104, 2005.

[13] I. V. Shadrivov, A. A. Sukhorukov, and Y. S. Kivshar, Guided modes in negative-refractive-index waveguides, Phys. Rev. E 67, 057602, 2003.

[14]A. K. Iyer, P. C. Kremer, and G. V. Eleftheriades, Experimental and theoretical verification of focusing in a large, periodically loaded transmission line negative refractive index metamaterial, Opt. Express 11(7): 696708, 2003.

[15] A. Grbic, and G. V. Eleftheriades, Periodic analysis of a 2-D negative refractive index transmission line structure, IEEE Trans. Antennas Propag. 51(10): 26042611, 2003. 